\title{
YIELD CONDITION FOR CONCRETE UNDER MODERATE HYDROSTATIC PRESSURE ${ }^{1}$
}

\author{
Aleksander Szwed, Inez Kamińska \\ Warsaw University of Technology, Institute of Building Engineering, Warsaw, Poland \\ e-mail: a.szwed@il.pw.edu.pl; ikam@il.pw.edu.pl
}

\begin{abstract}
A yield condition for concrete under moderate hydrostatic pressure is proposed as a nineparameter extension of the Lubliner yield criterion. The modification is introduced to both meridional and deviatoric cross-sections. Singularities are eliminated by means of a regularisation parameter of a clear geometrical interpretation. Calibration is carried out resulting in analytical formulae for the parameters depending on experimental data for five characteristic tests. A comparison with experimental data available in literature is drawn, showing consistency between the yield criterion and the test results. Convexity requirements for the proposed function are determined, leading to limitations on the input data.
\end{abstract}

Keywords: yield condition, concrete, Lubliner condition, concrete damaged plasticity

\section{Introduction}

Designing of yield conditions and plastic potentials suitable for concrete is an outgoing research. Two significant aspects are to be taken into consideration in the process. The yield condition should fit experimental results (Kupfer et al., 1969; Mills and Zimmerman, 1970; Gabet et al., 2008), which suggest several key features to be reproduced by theoretical models (Ottosen, 1977; Bigoni and Piccolroaz, 2004). The accurate description of the data requires quite complex functions. On the other hand, clarity or even simplicity is desired, especially when it comes to interpretation of material parameters and further numerical implementation. A proper yield function for concrete ensures keeping the balance between those two conditions. In this paper, we introduce an extension of the Lubliner yield function, which improves its flexibility, leaving the overall shape of the yield surface unchanged.

Experimental data indicate that continuous and convex functions of three independent invariants of the stress tensor properly describe the appearance and development of plastic flow. However, the data are not conclusive on differentiability, some singular points or lines are allowable (Bigoni and Piccolroaz, 2004). Generally, the assumption of isotropic behaviour is valid for concrete at failure. As to the specifics, the shape of meridians is non-linear and often modelled by a parabola or a hyperbola, although it is not possible to accurately describe the whole range of available experimental results by a single second-order curve. Moreover, the deviatoric cross-section of the yield surface tends to change from nearly triangular to nearly circular as the confinement increases (Ottosen, 1977).

The mechanism of failure of concrete strongly depends on the state of stress, more precisely on the signs of principal stresses (Kupfer et al., 1969; Mills and Zimmerman, 1970). For the states of pure tension $\left(\sigma_{3}>0\right)$ and mixed tension and compression $\left(\sigma_{1} \sigma_{3}<0\right)$, the failure occurs by cleavage fracture. The main crack is perpendicular to the direction of the maximum principal stress $\sigma_{1}$. In the case of pure compression $\left(\sigma_{1}<0\right)$, shear decohesion is the primary

${ }^{1}$ This work is related to a paper presented at PCM-CMM-2019. 
cause of destruction of the material. The mode of fracture is mixed when the stress state is near the points of $\sigma_{1}=0$. Thus, the equation $\sigma_{1}=0$ establishes the transition zone between those two mechanisms. It is evident that the two types of behaviour should be reflected in the yield and failure criteria. Basically, it can be done by combining two surfaces of different slopes in the meridional planes, which is the idea used by Lubliner et al. (1989).

The proposed yield condition is a regularisation of the strength criterion of Lubliner (Lubliner et al., 1989; Abaqus 6.11 Theory Manual, 2011) consisting of two cones with almost triangular deviatoric cross-sections in the Haigh-Westergaard stress space. The two-surface criterion with six parameters introduced by Lubliner allows for separate shaping of the yield condition in the triaxial compression zone and in the zone where at least one principal stress is positive, which complies with experimental data, as mentioned above. In this way, material hardening/softening can be conveniently incorporated into the model independently for both zones, using a simple analytical calibration process. Those advantages are preserved in our modification of the condition, but calibration becomes more complicated due to introduction of several new parameters. The Lubliner yield function possesses discontinuities of gradient on the curve of the null maximum principal stress and along tensile and compressive meridians. Those unfavourable features result from non-smooth intersection of two cones and the applied singular deviatoric shape functions. At the expense of an increase of the number of parameters, in the presented yield criterion the singularities are eliminated with the exception of the apex of the yield surface.

In order to remove the singularities of the yield function gradient along the tensile and compressive meridians, smooth shape functions and an additional regularisation parameter, specifically responsible for smoothening the surface in the vicinity of the null maximum principal stress curve, are introduced. One of the classical most widely recognised shape function proposed by Ottosen (1977) and its later generalisation by Podgórski (1984) are considered. As mentioned, the singularity at the apex (equal triaxial tension) remains. This disadvantage can be removed as well by the employment of a second regularisation parameter (Szwed and Kamińska, 2017), but it is not presented here.

In the following Sections, we describe the proposed criterion and show its graphical representation. Calibration of the model being focused on application in the plasticity and damage theory is discussed extensively, also including restrictions resulting from convexity requirements. Closed formulae for nine (or seven for a simplified version) parameters are derived, suitable for introduction of hardening/softening functions. We check the consistency of the criterion prediction with available empirical data for concrete. Comparison with similar yield criteria is carried out in order to show the flexibility of the proposed surface.

\section{Definition of the modified yield condition}

The cylindrical invariants $\xi, r$ and $\Theta$ of the stress tensor $\boldsymbol{\sigma}$ are defined as

$$
\xi=\frac{1}{\sqrt{3}} \operatorname{tr} \boldsymbol{r} \quad \boldsymbol{\sigma}=\|\mathbf{s}\|=\sqrt{\operatorname{tr}^{2}} \quad \Theta=\frac{1}{3} \arccos \frac{\sqrt{6} \operatorname{tr} \mathbf{s}^{3}}{\sqrt{\operatorname{tr}^{3} \mathbf{s}^{2}}}
$$

and $\mathbf{s}=\boldsymbol{\sigma}-\xi \mathbf{k}, \mathbf{k}=\mathbf{I} / \sqrt{3}$, where $\mathbf{I}$ is the second order unit tensor and $\operatorname{tr}$ denotes trace of a second order tensor.

We propose the following yield criterion

$$
f(\xi, r, \Theta)=2 \xi+\sqrt{4 B^{2}+\left[\left(\beta_{1}-\alpha_{1} g r\right)-\left(\beta_{2}-\alpha_{2} h r\right)\right]^{2}}-\left(\beta_{1}-\alpha_{1} g r\right)-\left(\beta_{2}-\alpha_{2} h r\right)=0
$$

uniquely separating the safe from unsafe stress states. $\alpha_{1}, \alpha_{2}, \beta_{1}, \beta_{2}$ and $B$ are material parameters, whereas $g$ and $h$ are deviatoric section shape functions of Podgórski (1984) 


$$
g(\Theta)=\cos \frac{\arccos \left(\gamma_{1} \cos 3 \Theta\right)-\arccos \delta_{1}}{3} \quad h(\Theta)=\cos \frac{\arccos \left(\gamma_{2} \cos 3 \Theta\right)-\arccos \delta_{2}}{3}
$$

with values in the range $1 / 2 \leqslant g(\Theta) \leqslant 1$ and $1 / 2 \leqslant h(\Theta) \leqslant 1$. When $g(\Theta) \equiv 1$ and $h(\Theta) \equiv 1$, a rotational surface consisting of combined two Drucker-Prager (1952) cones is obtained. For $\delta_{1}=\delta_{2}=1$, the shape functions of Ottosen (1977) are retrieved. If $\gamma_{1}=\gamma_{2}=1$, the generalised shape functions for the Coulomb-Mohr condition are derived, and the surface defined by Eq. (2.2) has edges along the tensile, shear and compressive meridians, compare Szwed and Kamińska (2019).

Functions $g$ and $h$ dependent on the Lode angle $\Theta$ determine the shape of deviatoric crosssections of the yield locus. The first function describes the deviatoric shape predominately in the tension zone with at least one principal stress positive, whereas the second rules the compression zone with all principal stresses negative. As a result, the curve $f(\xi, r, \Theta)=0$ for fixed $\xi$ is periodic of period $2 \pi / 3$ with the axes of symmetry described by $\Theta=k \pi / 3$ for $k=0,1,2$. Invariant $\cos 3 \Theta$ is used in the definition of the shape functions to preserve those symmetries (Jemioło and Szwed, 1999).

Parameters $\alpha_{1}, \beta_{1}, \gamma_{1}, \delta_{1}$ and $\alpha_{2}, \beta_{2}, \gamma_{2}, \delta_{2}$ are material constants associated with the tension and compression cone, accordingly

$$
\xi+\alpha_{1} r g(\Theta)-\beta_{1}=0 \quad \xi+\alpha_{2} r h(\Theta)-\beta_{2}=0
$$

Depending on the assumed form of the shape functions, eight (for the Podgórski shape functions) or six (for the Ottosen shape functions) parameters are determined using typical experimental tests. The role of the ninth (seventh) parameter $B$ in Eq. (2.2) is to smoothen the intersection zone of those cones. Its value here is chosen arbitrarily, but can be determined via a curve fitting technique.

Function (2.2) is convex if the meridians are convex, and the following requirements for convexity of positive shape functions $g$ and $h$ are met (Bigoni and Piccolroaz, 2004)

$$
g(\Theta)+g^{\prime \prime}(\Theta) \geqslant 0 \quad \text { and } \quad h(\Theta)+h^{\prime \prime}(\Theta) \geqslant 0
$$

Based on those conditions, the following inequalities have to be satisfied for the material parameters in functions $(2.3)$

$$
\begin{array}{lcc}
\alpha_{2} \geqslant \alpha_{1}>0 & \beta_{2} \geqslant \beta_{1}>0 \\
\left|\gamma_{2}\right| \leqslant 1 & \left|\delta_{2}\right| \leqslant 1 \quad\left|\gamma_{1}\right| \leqslant 1
\end{array} \quad\left|\delta_{1}\right| \leqslant 1
$$

\section{Calibration of parameters}

Material parameters $\alpha_{1}, \beta_{1}, \gamma_{1}, \delta_{1}$ and $\alpha_{2}, \beta_{2}, \gamma_{2}, \delta_{2}$ are calibrated based on five commonly used experimental tests listed in Table 1. Two of them are located on the tensile meridian $(\Theta=0)$, namely the uniaxial tension test and the equibiaxial compression test, the next two - on the compressive meridian $(\Theta=\pi / 3)$ - the uniaxial compression test and the triaxial compression test and the last one on the shear meridian $(\Theta=\pi / 6)$. In the case of Ottosen or Coulomb-Mohr criteria, only four tests are needed. Parameter $B$ is left to be chosen arbitrarily with specification of recommended values.

The intersection curve of two cones (2.4) is defined by the condition

$$
\alpha_{1} g r_{K}-\beta_{1}=\alpha_{2} h r_{K}-\beta_{2}
$$


Table 1. Experimental tests used for calibration

\begin{tabular}{|c|c|c|c|c|c|}
\hline $\begin{array}{c}\text { Principal } \\
\text { stresses, } \\
\text { invariants }\end{array}$ & $\begin{array}{c}\text { Uniaxial } \\
\text { tension } \\
\sigma_{T}\end{array}$ & $\begin{array}{c}\text { Uniaxial } \\
\text { compression } \\
\sigma_{C}\end{array}$ & $\begin{array}{c}\text { Biaxial } \\
\text { compression } \\
\sigma_{B C}\end{array}$ & $\begin{array}{c}\text { Triaxial } \\
\text { compression } \\
\sigma_{T C}, \eta>1\end{array}$ & $\begin{array}{c}\text { Biplane } \\
\text { shear } \\
\sigma_{B S}\end{array}$ \\
\hline \hline$\sigma_{1}$ & $\sigma_{T}$ & 0 & 0 & $-\sigma_{T C}$ & 0 \\
\hline$\sigma_{2}$ & 0 & 0 & $-\sigma_{B C}$ & $-\sigma_{T C}$ & $-\sigma_{B S} / 2$ \\
\hline$\sigma_{3}$ & 0 & $-\sigma_{C}$ & $-\sigma_{B C}$ & $-\eta \sigma_{T C}$ & $-\sigma_{B S}$ \\
\hline$\xi$ & $\xi_{T}=\frac{\sigma_{T}}{\sqrt{3}}$ & $\xi_{C}=-\frac{\sigma_{C}}{\sqrt{3}}$ & $\xi_{B C}=-\frac{2 \sigma_{B C}}{\sqrt{3}}$ & $\xi_{T C}=-\sigma_{T C} \frac{2+\eta}{\sqrt{3}}$ & $\xi_{B S}=-\sigma_{B S} \frac{\sqrt{3}}{2}$ \\
\hline$r$ & $r_{T}=\sigma_{T} \sqrt{\frac{2}{3}}$ & $r_{C}=\sigma_{C} \sqrt{\frac{2}{3}}$ & $r_{B C}=\sigma_{B C} \sqrt{\frac{2}{3}}$ & $r_{T C}=\sigma_{T C}(\eta-1) \sqrt{\frac{2}{3}}$ & $r_{B S}=\sigma_{B S} \frac{1}{\sqrt{2}}$ \\
\hline$\Theta$ & 0 & $\pi / 3$ & 0 & $\pi / 3$ & $\pi / 6$ \\
\hline
\end{tabular}

which used in Eq. (2.2) leads to the equation

$$
2\left(\xi_{K}+B\right)-\left(\beta_{1}-\alpha_{1} g r_{K}\right)-\left(\beta_{2}-\alpha_{2} h r_{K}\right)=0
$$

Solution to the set of Eqs. (3.1) and (3.2) is a parametric definition of the following curve

$$
r_{K}(\Theta)=\frac{\beta_{2}-\beta_{1}}{\alpha_{2} h-\alpha_{1} g} \quad \xi_{K}(\Theta)=\frac{\beta_{1} \alpha_{2} h-\beta_{2} \alpha_{1} g}{\alpha_{2} h-\alpha_{1} g}-B
$$

where angle $\Theta$ is the parameter. For $B=0$, the formulae define the intersection curve for cones (2.4) denoted as $r_{K C}(\Theta), \xi_{K C}(\Theta)$. Then, parameter $B$ has a clear geometrical interpretation shown in Fig. 1. In the meridional cross-section of the proposed surface $B$ defines a shift of curve (3.3) in the direction of invariant $\xi$ from the intersection curve of cones (2.4). Coordinates $r$ are the same for both curves.

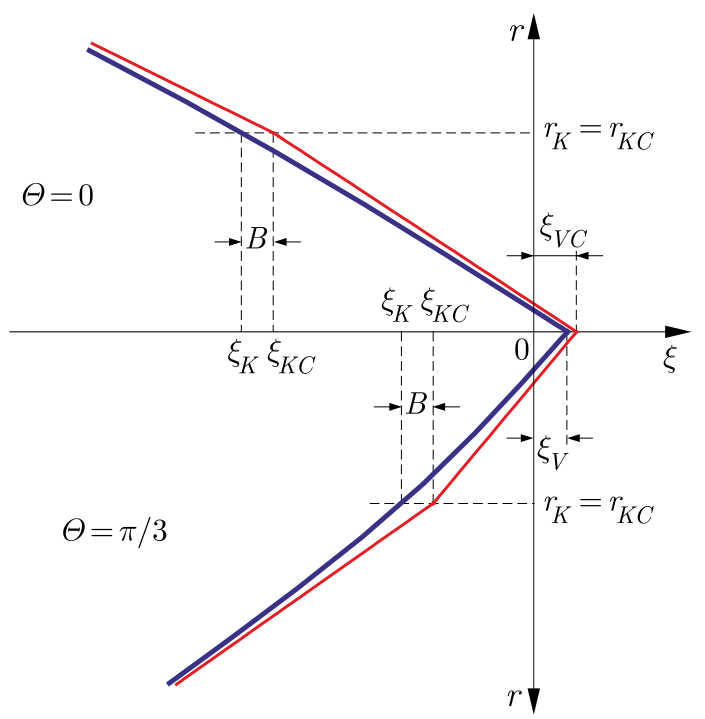

Fig. 1. Interpretation of $B$ parameter in the meridional cross-section of the yield surfaces

Using Eqs. (3.1) in (3.2) twice, the following results can be derived

$$
\xi_{K}+B=\beta_{1}-\alpha_{1} r_{K} g(\Theta) \quad \xi_{K}+B=\beta_{2}-\alpha_{2} r_{K} h(\Theta)
$$

Equations (3.4) are very convenient for calibration of the free parameters. It is assumed that experimental tests carried out in the plane stress conditions are located on curve (3.3), so at the 
same time Eqs. (3.4) are fulfilled. This feature allows one to obtain closed forms formulae for the searched parameters.

Denoting by $g_{T}, g_{C}, g_{S}$ and $h_{T}, h_{C}, h_{S}$ values of shape functions (2.3) for the characteristic meridians, we obtain the relations

$$
\begin{array}{rlrl}
g_{T} & =g(0)=\cos \left(\psi_{1}-\chi_{1}\right) & g_{S}=g\left(\frac{\pi}{3}\right) & =\cos \left(\frac{\pi}{6}-\chi_{1}\right) \\
g_{C} & =g\left(\frac{\pi}{3}\right)=\cos \left(\frac{\pi}{3}-\psi_{1}-\chi_{1}\right) & h_{T} & =h(0)=\cos \left(\psi_{2}-\chi_{2}\right) \\
h_{S} & =h\left(\frac{\pi}{6}\right)=\cos \left(\frac{\pi}{6}-\chi_{2}\right) & h_{C}=h\left(\frac{\pi}{3}\right)=\cos \left(\frac{\pi}{3}-\psi_{2}-\chi_{2}\right)
\end{array}
$$

where additional notations are introduced

$$
\psi_{1}=\frac{1}{3} \arccos \gamma_{1} \quad \psi_{2}=\frac{1}{3} \arccos \gamma_{2} \quad \chi_{1}=\frac{1}{3} \arccos \delta_{1} \quad \chi_{2}=\frac{1}{3} \arccos \delta_{2}
$$

Using Eqs. (3.5) and the ratios of the characteristic values of the shape functions defined as

$$
p=\frac{g_{T}}{g_{C}} \quad q=\frac{h_{T}}{h_{C}} \quad k=\frac{g_{T}}{g_{S}} \quad t=\frac{h_{T}}{h_{S}}
$$

where $g_{T}, g_{C}, g_{S}$ and $h_{T}, h_{C}, h_{S}$ are expressed via $p, q$ and $k, t$ parameters as follows

$$
\begin{aligned}
& g_{S}=\sqrt{\frac{4 p^{2}-k^{2}(1+p)^{2}}{4 p\left(p-k^{2}\right)}} \quad g_{T}=k g_{S} \quad g_{C}=\frac{k}{p} g_{S} \\
& h_{S}=\sqrt{\frac{4 q^{2}-t^{2}(1+q)^{2}}{4 q\left(q-t^{2}\right)}} \quad h_{T}=t h_{S} \quad h_{C}=\frac{t}{q} h_{S}
\end{aligned}
$$

and using trigonometric identities, the deviatoric cross-section shape parameters $\gamma_{1}, \gamma_{2}$ and $\delta_{1}, \delta_{2}$ can be found as functions of $p, q$ and $k, t$, i.e.

$$
\begin{array}{lc}
\gamma_{1}=\frac{k^{2}(1+p)^{2}-p^{2}}{2 p^{3}} \sqrt{4 p^{2}-k^{2}(1+p)^{2}} & \gamma_{2}=\frac{t^{2}(1+q)^{2}-q^{2}}{2 q^{3}} \sqrt{4 q^{2}-t^{2}(1+q)^{2}} \\
\delta_{1}=\frac{k(p-1)\left[3 p^{2}-k^{2}\left(p^{2}+p+1\right)\right]}{2 \sqrt{\left[p\left(p-k^{2}\right)\right]^{3}}} & \delta_{2}=\frac{t(q-1)\left[3 q^{2}-t^{2}\left(q^{2}+q+1\right)\right]}{2 \sqrt{\left[q\left(q-t^{2}\right)\right]^{3}}}
\end{array}
$$

Obtained results (3.9) are supposed to meet the following restrictions

$$
\begin{array}{lll}
1 \leqslant p \leqslant 2 & 1 \leqslant q \leqslant 2 & \text { and } \\
\frac{\sqrt{3} p}{1+p} \leqslant k \leqslant \frac{p}{\sqrt{p^{2}-p+1}} & \frac{\sqrt{3} q}{1+q} \leqslant t \leqslant \frac{q}{\sqrt{q^{2}-q+1}}
\end{array}
$$

which are equivalent to convexity requirements (2.6) limited to

$$
0 \leqslant \gamma_{1} \leqslant 1 \quad 0 \leqslant \gamma_{2} \leqslant 1 \quad \text { and } \quad 0 \leqslant \delta_{1} \leqslant 1 \quad 0 \leqslant \delta_{2} \leqslant 1
$$

The limits on $p$ and $q$ parameters follow their range reported in experiments for concrete and frictional materials (Ottosen, 1977; Podgórski, 1984). Graphical representation of restrictions (3.10) as shaded regions and contours for parameters $\gamma_{1}, \gamma_{2}, \delta_{1}, \delta_{2}$ according to Eqs. (3.9) are shown in Fig. 2. 

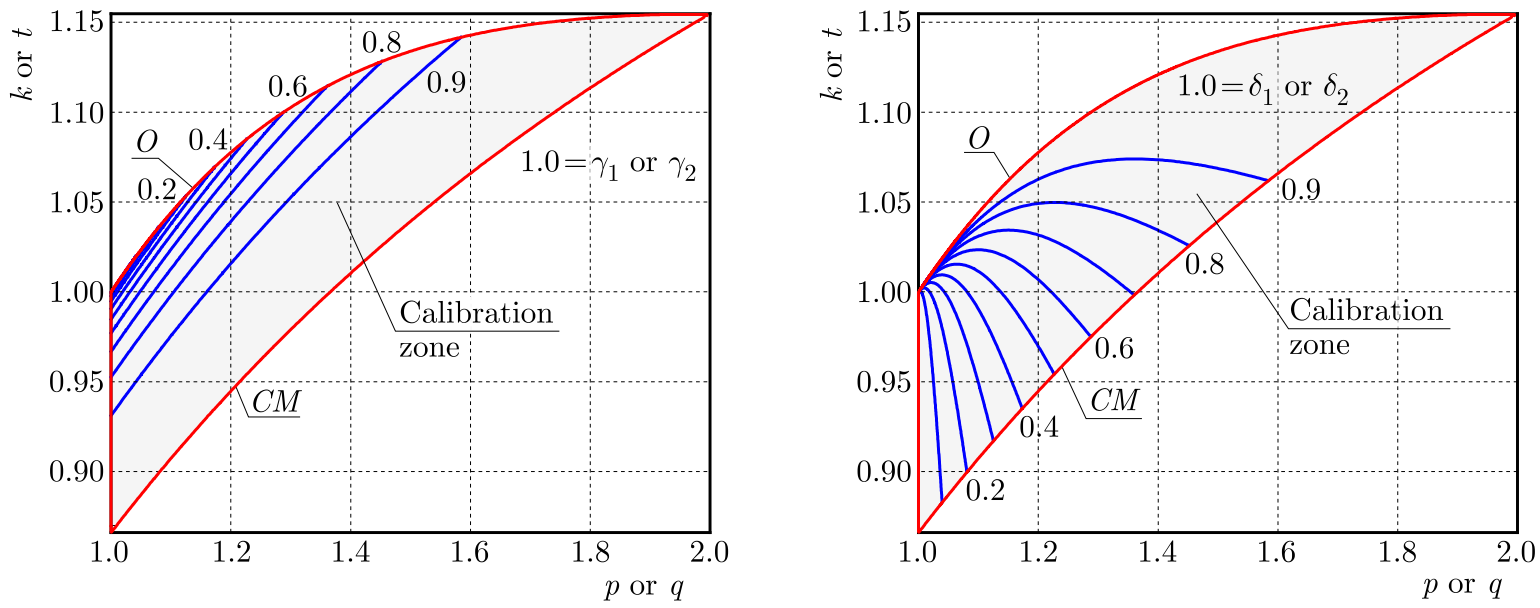

Fig. 2. Limits for parameters $p, q, k, t$ due to convexity requirements, where $O$ stands for Ottosen and $C M$ for Coulomb-Mohr shape functions

Using values of the stress invariants from Table 1, notation (3.7) in relations (3.4), the following can be obtained

$$
\begin{aligned}
& \alpha_{1}=\frac{\xi_{C}-\xi_{B C}}{g_{C}\left(p r_{B C}-r_{C}\right)} \quad \beta_{1}=B+\frac{p \xi_{C} r_{B C}-\xi_{B C} r_{C}}{p r_{B C}-r_{C}} \\
& \alpha_{2}=\frac{\xi_{C}-\xi_{B C}}{h_{C}\left(q r_{B C}-r_{C}\right)} \quad \beta_{2}=B+\frac{q \xi_{C} r_{B C}-\xi_{B C} r_{C}}{q r_{B C}-r_{C}} \\
& k=\frac{p r_{B S}\left(\xi_{C}-\xi_{B C}\right)}{p r_{B C}\left(\xi_{C}-\xi_{B S}\right)+r_{C}\left(\xi_{B S}-\xi_{B C}\right)} \quad t=\frac{q r_{B S}\left(\xi_{C}-\xi_{B C}\right)}{q r_{B C}\left(\xi_{C}-\xi_{B S}\right)+r_{C}\left(\xi_{B S}-\xi_{B C}\right)}
\end{aligned}
$$

The parameters $p$ and $q$ are still undetermined.

Eliminating the square root, Eq. (2.2) can be transformed into the form

$$
\left[\alpha_{1} g r-\left(\beta_{1}-\xi\right)\right]\left[\alpha_{2} h r-\left(\beta_{2}-\xi\right)\right]=B^{2}
$$

which is used for calibration of the two remaining parameters. One of roots of this quadratic equation defines criterion (2.2). Application of Eq. (3.13) to the cases of uniaxial tension and triaxial compression tests yields the following formulae for parameters

$$
\begin{array}{r}
p=\frac{\beta_{T} \lambda_{C}-\beta_{C} \lambda_{T}-\sqrt{\left(\beta_{T} \lambda_{C}-\beta_{C} \lambda_{T}\right)^{2}+4\left(\beta_{C} \delta_{T}+\beta_{T} \delta_{C}\right)\left(\delta_{C} \lambda_{T}+\delta_{T} \lambda_{C}\right)}}{2\left(\beta_{C} \delta_{T}+\beta_{T} \delta_{C}\right)} \\
q=\frac{\beta_{T} \lambda_{C}-\beta_{C} \lambda_{T}+\sqrt{\left(\beta_{T} \lambda_{C}-\beta_{C} \lambda_{T}\right)^{2}+4\left(\beta_{C} \delta_{T}+\beta_{T} \delta_{C}\right)\left(\delta_{C} \lambda_{T}+\delta_{T} \lambda_{C}\right)}}{2\left(\beta_{C} \delta_{T}+\beta_{T} \delta_{C}\right)}
\end{array}
$$

with additional notations

$$
\begin{array}{lll}
\beta_{T}=a_{T}\left(a_{T}-2 B r_{B C}\right) & \delta_{T}=B\left(a_{T} r_{C}+b_{T} r_{B C}\right)-a_{T} b_{T} & \lambda_{T}=b_{T}\left(b_{T}-2 B r_{C}\right) \\
\beta_{C}=b_{C}\left(b_{C}+2 B r_{B C}\right) & \delta_{C}=B\left(b_{C} r_{C}+a_{C} r_{B C}\right)+a_{C} b_{C} & \lambda_{C}=a_{C}\left(a_{C}+2 B r_{C}\right)
\end{array}
$$

and

$$
\begin{array}{lr}
a_{T}=\left(\xi_{C}-\xi_{B C}\right) r_{T}+\left(\xi_{T}-\xi_{C}\right) r_{B C} & b_{T}=\left(\xi_{T}-\xi_{B C}\right) r_{C} \\
a_{C}=\left(\xi_{B C}-\xi_{T C}\right) r_{C}+\left(\xi_{C}-\xi_{B C}\right) r_{T C} & b_{C}=\left(\xi_{C}-\xi_{T C}\right) r_{B C}
\end{array}
$$

As an option, calibration simplified compared to (3.14) can be regarded. The parameter $q$ is assumed or calculated from Eq. (3.13) for $B=0$, which means that the point representing 
the triaxial compression test lays in the vicinity of the yield surface (but does not belong to it exactly). This assumption yields

$$
q_{S}=\frac{\left(\xi_{B C}-\xi_{T C}\right) r_{C}+\left(\xi_{C}-\xi_{B C}\right) r_{T C}}{\left(\xi_{C}-\xi_{T C}\right) r_{B C}} \quad \text { then } \quad p_{S}=-\frac{\delta_{T} q_{S}+\lambda_{T}}{\beta_{T} q_{S}+\delta_{T}}
$$

Notations (3.15) are still valid and results (3.12) are the same.

\section{Results and discussion}

Eight material parameters $\alpha_{1}, \beta_{1}, \gamma_{1}, \delta_{1}$ and $\alpha_{2}, \beta_{2}, \gamma_{2}, \delta_{2}$ are calculated based on five tests as described in the previous Section, whereas the parameter $B$ is arbitrary. Typical strength ratios for concrete available in literature (Kupfer et al., 1969; Mills and Zimmerman, 1970; Gabet et al., 2008) are

$$
\begin{array}{lcc}
\sigma_{T}=0.1 \sigma_{C} & \sigma_{B C}=1.16 \sigma_{C} & \sigma_{B S}=1.28 \sigma_{C} \\
\sigma_{T C}=1.25 \sigma_{C} & \eta & =4.91
\end{array}
$$

The data for $\sigma_{T C}$ and $\eta$ can be estimated from various experiments (various locations of $\xi_{T C}$ ) and the results can differ significantly, which is discussed in the second part of this Section. The yield limit in the uniaxial compression test $\sigma_{C}$ is treated as a scaling factor in the following graphical presentations of the results. Calculations for several values of $B=\alpha_{B} \sigma_{T}=0.1 \alpha_{B} \sigma_{C}$ are performed. The obtained values of the parameters are presented in Table 2 .

Inevitably, a change in the value of $B$ influences the whole yield surface. Although, as it turns out, the impact on the tension cone (on $\alpha_{1}, \beta_{1}, \gamma_{1}, \delta_{1}$ ) is greater than on the compression cone (on $\alpha_{2}, \beta_{2}, \gamma_{2}, \delta_{2}$ ). Qualitatively, the increase of $B$ implies "sharpening" of the corners of the rounded hexagonal deviatoric section in the tension zone, whereas "blunting" of the corners in the compression zone. The surface vertex changes its location $\xi_{V}=\left[\left(\beta_{1}+\beta_{2}\right)-\sqrt{4 B^{2}+\left(\beta_{2}-\beta_{1}\right)^{2}}\right] / 2$ (see Fig. 1) with a change of $B$ as well. We recommend rather small values of $B$, which limits its influence on the shape of the yield surface to the transition zone and the location of the apex.

Table 2. Material parameters for typical experimental data (4.1) and the assumed $B$

\begin{tabular}{|c|c|c|c|c|c|}
\hline Parameter & $B=0$ & $B=0.5 \sigma_{T}$ & $B=\sigma_{T}$ & $B=1.5 \sigma_{T}$ & $B=2 \sigma_{T}$ \\
\hline \hline$p$ & 1.7188 & 1.7757 & 1.8263 & 1.8724 & 1.9154 \\
\hline$k$ & 1.1256 & 1.1321 & 1.1376 & 1.1424 & 1.1467 \\
\hline$\gamma_{1}$ & 0.9884 & 0.9932 & 0.9962 & 0.9980 & 0.9992 \\
\hline$\delta_{1}$ & 0.9902 & 0.9943 & 0.9968 & 0.9984 & 0.9993 \\
\hline$\alpha_{1}$ & 1.6144 & 1.5639 & 1.5240 & 1.4912 & 1.4632 \\
\hline$\beta_{1} / \sigma_{C}$ & 0.1895 & 0.1917 & 0.2040 & 0.2229 & 0.2464 \\
\hline$q$ & 1.4412 & 1.4350 & 1.4291 & 1.4236 & 1.4184 \\
\hline$t$ & 1.0881 & 1.0871 & 1.0862 & 1.0853 & 1.0845 \\
\hline$\gamma_{2}$ & 0.9307 & 0.9283 & 0.9261 & 0.9239 & 0.9218 \\
\hline$\delta_{2}$ & 0.9376 & 0.9354 & 0.9333 & 0.9312 & 0.9292 \\
\hline$\alpha_{2}$ & 2.0024 & 2.0155 & 2.0280 & 2.0400 & 2.0516 \\
\hline$\beta_{2} / \sigma_{C}$ & 0.5570 & 0.6194 & 0.6813 & 0.7426 & 0.8036 \\
\hline
\end{tabular}

Results of the simplified calibration based on Eqs. (3.17) are presented in Table 3. For the compression zone, $\alpha_{2}, \beta_{2}, \gamma_{2}, \delta_{2}$ are calculated as for cone $(2.4)_{2}$. Then $\alpha_{1}, \beta_{1}, \gamma_{1}$ and $\delta_{1}$ are computed for the assumed value of $B$. The estimated values are the following: $q_{S}=1.4412$, $t_{S}=1.0881$, then $\gamma_{2}=0.9307, \delta_{2}=0.9376, \alpha_{2}=2.0024$ and $\beta_{2}=0.5570 \sigma_{C}$ regardless of $B$, and 
the remaining parameters are given in Table 3 . Note that for $B \cong \sigma_{T}=0.1 \sigma_{C}$, the parameters are obtained with an error of the order $0.2 \%$ comparing to Table 2 . This approach to calibration can be successfully used in practice.

Table 3. Material parameters for typical data and the assumed $B$ for simplified calibration

\begin{tabular}{|c|c|c|c|c|c|}
\hline Parameter & $B=0$ & $B=0.5 \sigma_{T}$ & $B=\sigma_{T}$ & $B=1.5 \sigma_{T}$ & $B=2 \sigma_{T}$ \\
\hline \hline$p$ & 1.7188 & 1.7755 & 1.8246 & 1.8675 & 1.9054 \\
\hline$k$ & 1.1256 & 1.1321 & 1.1374 & 1.1419 & 1.1457 \\
\hline$\gamma_{1}$ & 0.9884 & 0.9932 & 0.9961 & 0.9979 & 0.9990 \\
\hline$\delta_{1}$ & 0.9902 & 0.9943 & 0.9967 & 0.9982 & 0.9992 \\
\hline$\alpha_{1}$ & 1.6144 & 1.5641 & 1.5253 & 1.4945 & 1.4795 \\
\hline$\beta_{1} / \sigma_{C}$ & 0.1895 & 0.1919 & 0.2052 & 0.2261 & 0.2524 \\
\hline
\end{tabular}

The proposed yield condition is not able to accommodate every set of five test results because of the cosine and arccosine expressions appearing in the shape functions, which put restraints on their arguments as well as on their values. It is possible that the yield surface cannot be "stretched" or "bent" to pass through the points representing one or more of the calibrating tests. The limits on parameters must be carefully checked due to convexity requirements (2.6), since those conditions can be violated for given experimental data or the assumed value of $B$. Then formulae (3.12), (3.14)-(3.17) can produce imaginary numbers, which is impracticable. For example, in Fig. 3, a graph of function $p(B)$ is shown, which passes through the limit value $p=2$ when $B=0.30625 \sigma_{C}$. Therefore, the convexity condition for the given data is violated for $B>0.30625 \sigma_{C}$. The shape parameter $k(B)$ is presented in the second graph with the upper and lower limits set in Eqs. (3.10), with the exactly same value of $B$ causing loss of convexity. In the third graph, the shape parameter $t(B)$ is shown exhibiting no problems with convexity in the given range of $B$.

However, for typical proportions of the yield limits for concrete and $B \sim 0.2 \sigma_{C}$ (and lower), the convexity conditions are usually met, when the calibrating triaxial test of a moderate $\xi_{T C}$ is chosen. Primarily, the restraints apply to uncommon ratios $\alpha_{T}=\sigma_{T} / \sigma_{C}, \alpha_{B C}=\sigma_{B C} / \sigma_{C}$, $\alpha_{B S}=\sigma_{B S} / \sigma_{C}$ or $\alpha_{T C}=\sigma_{T C} / \sigma_{C}$ (and $\eta$ ), which can occur during kinematic hardening.
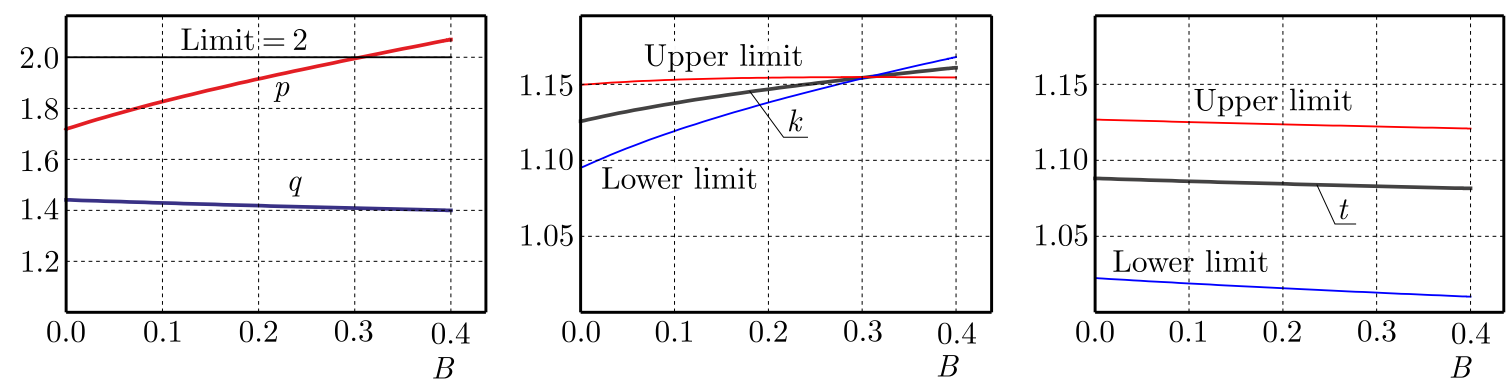

Fig. 3. Dependence of $p, q$ and $k, t$ on the assumed values of $B$

Three-dimensional views of the criterion in the principal stresses and the three-dimensional representation of the surface for the plane stress in the stress component space are shown in Fig. 4.

In Fig. 5, the characteristic meridians are shown along with the points used in calibration as well as the deviatoric and plane stress cross-sections. A change in the meridian slope is visible when passing through the transition zone (the curve containing $\sigma_{C}, \sigma_{B C}$ and $\sigma_{B S}$, see Fig. 4). The distinction between the two cones is clear for all the presented meridians, although it is most significant for the compressive meridian. The shape of the deviatoric cross-section changes from nearly triangular, with sharp corners for a low confinement, to rounded hexagons for a 

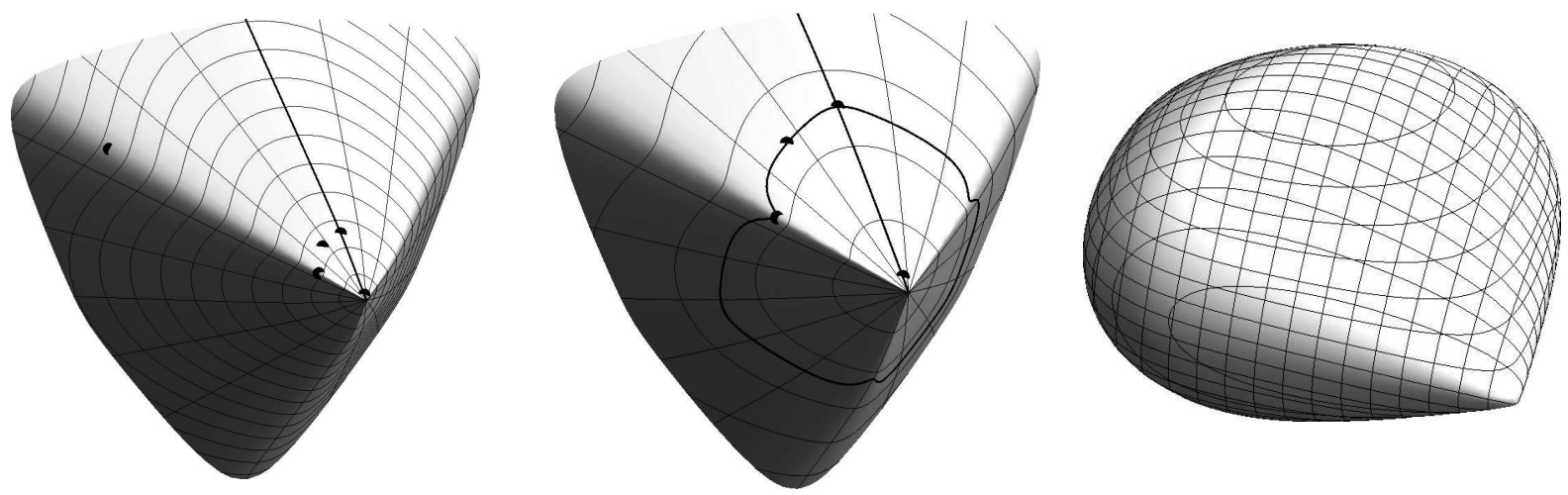

Fig. 4. Three-dimensional view of the strength criterion with marked calibration points in the principal stresses (left), apex zone with calibration curve (central), and for the plane stress in components (right), $B=\sigma_{T}=0.1 \sigma_{C}$
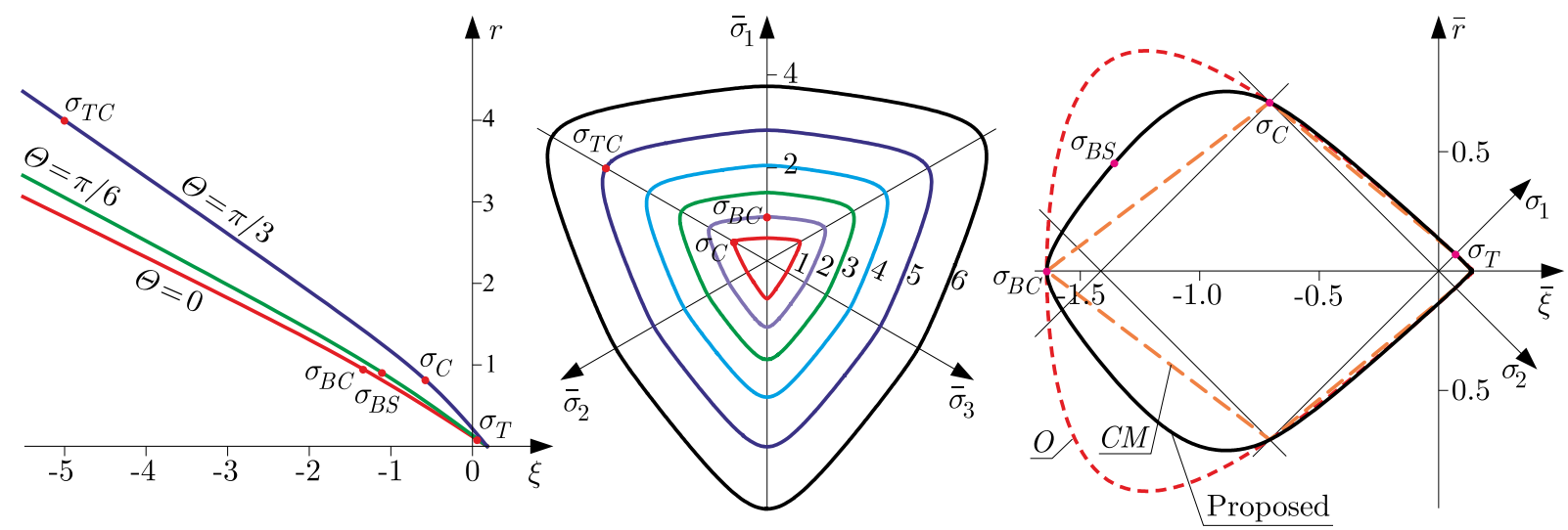

Fig. 5. Cross-sections of the yield surface: tensile, shear and compressive meridians with marked calibration points (left); shape of deviatoric cross-sections of the plasticity surface for $\xi$ equal to: 1) $\xi_{C}$, 2) $\left.\left.\left.\left.\xi_{B C}, 3\right) 4 \xi_{C}, 4\right) 6 \xi_{C}, 5\right) \xi_{T C}, 6\right) 12 \xi_{C}, \bar{\sigma}_{i}=\sigma_{i} \sqrt{2 / 3}$ (centre); the plane stress yield curve $\left(\sigma_{3}=0\right)$ in comparison with criteria based on the Ottosen and Coulomb-Mohr shape functions (right),

$$
\sqrt{2} \bar{\xi}=\sigma_{1}+\sigma_{2}, 2 \bar{r}^{2}=\left(\sigma_{1}-\sigma_{2}\right)^{2}, B=\sigma_{T}=0.1 \sigma_{C}
$$

high confinement. The proposed plane stress yield curve $\left(\sigma_{3}=0\right)$ is located between the limiting curves for the Ottosen and Coulomb-Mohr shape functions in the transition zone.

Next, in Fig. 6, cross-sections of the plasticity surface for constant values of one principal stress are presented. The curves illustrate basic features of the criterion when it changes from tensile to compressive zones.

The choice of the exact location of the triaxial compression test used in calibration influences strongly the overall shape of the yield surface, particularly the compressive cone, i.e. slope $\alpha_{2}$ and $\beta_{2}, \gamma_{2}, \delta_{2}$, see Table 4 . Obviously, it has a considerable impact on the plane stress cross-section as it is depicted in Fig. 7. Choosing triaxial tests of lower $\xi$ smoothens the curves in the biaxial compression zone.

Comparison with the experimental data of Kupfer et al. (1969), Mills and Zimmerman (1970) and Gabet et al. (2008) indicates that for a low-compression regime, it is suitable to use the triaxial test located at $\xi_{T C} \sim(-4 \div-2) \sigma_{C}$, whereas for a high-compression zone, it is appropriate to use a test corresponding to a much lower $\xi_{T C}$. From the available data, we recommend to use the following values of the parameters: $\alpha_{T C}=0.2600$ and $\eta=9.4976\left(\xi_{T C}=-1.726 \sigma_{C}\right)$ for the low-compression zone, but $\alpha_{T C}=12.3778$ and $\eta=2.8555\left(\xi_{T C}=-34.670 \sigma_{C}\right)$ for the high-compression zone, see Figs. 8 and 9. Then the adjustment of the meridians is best. For the planestress cross section, the last recommended set of values is the most suitable $\left(\xi_{T C}=-34.670 \sigma_{C}\right)$, although the other triaxial test also complies with the experimental data, see Fig. 10. 

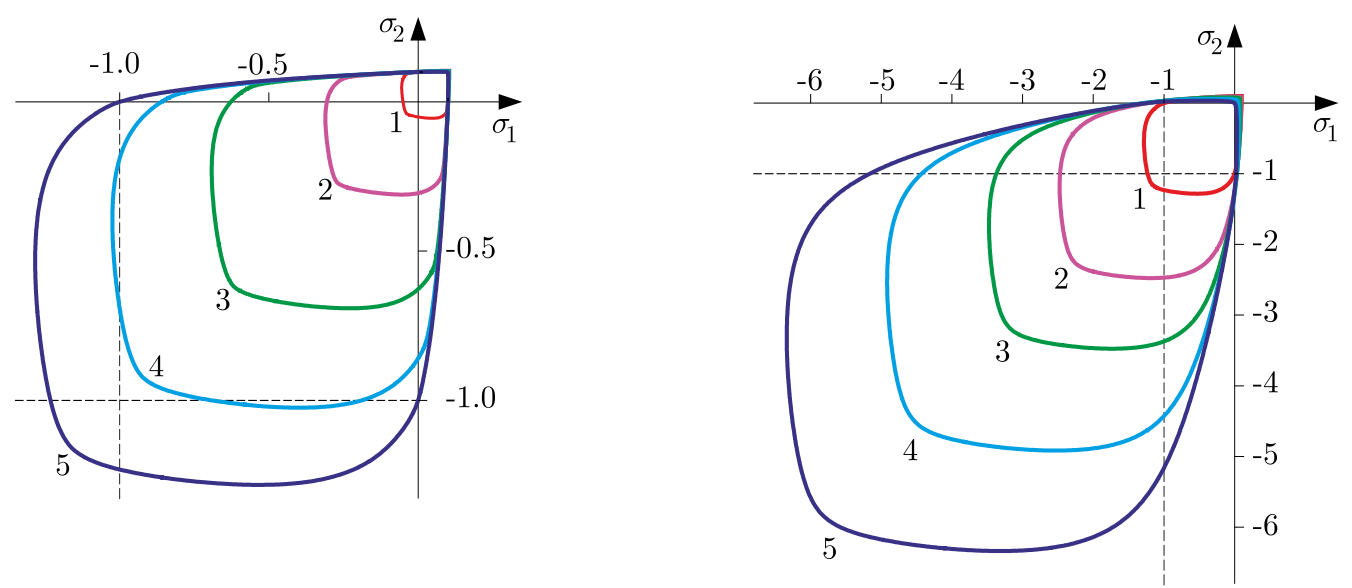

Fig. 6. Plasticity surface cross-sections for fixed $\sigma_{3}$ for tension zone (left): 1) $\left.\sigma_{3}=0.98 \sigma_{T}, 2\right) 0.85 \sigma_{T}$, 3) $\left.\left.0.6 \sigma_{T}, 4\right) 0.3 \sigma_{T}, 5\right) 0$; and compression zone (right): 1) 0,2$\left.\left.\left.)-0.2 \sigma_{C}, 3\right)-0.4 \sigma_{C}, 4\right)-0.7 \sigma_{C}, 5\right)-\sigma_{C}$,

$$
B=\sigma_{T}=0.1 \sigma_{C}
$$

Table 4. Material parameters for typical data: $\alpha_{T}=0.1, \alpha_{B C}=1.16, \alpha_{B S}=1.25$ and the assumed $B=0.2 \sigma_{C}$ for various locations of the triaxial compression test (Mills and Zimmerman, 1970; Gabet et al., 2008)

\begin{tabular}{|c|c|c|c|c|c|c|c|c|c|c|}
\hline$\eta$ & $\alpha_{T C}$ & $\xi_{T C} / \sigma_{C}$ & $\alpha_{1}$ & $\alpha_{2}$ & $\beta_{1} / \sigma_{C}$ & $\beta_{2} / \sigma_{C}$ & $\gamma_{1}$ & $\delta_{1}$ & $\gamma_{2}$ & $\delta_{2}$ \\
\hline \hline 2.8555 & 12.378 & -34.700 & 1.4331 & 2.4578 & 0.2179 & 1.1849 & 0.9999 & 0.9995 & 0.8909 & 0.8052 \\
\hline 2.8347 & 6.7222 & -18.734 & 1.4304 & 2.5214 & 0.2153 & 1.2447 & 0.9999 & 0.9995 & 0.8828 & 0.7886 \\
\hline 4.7959 & 1.0889 & -4.2724 & 1.4567 & 2.1116 & 0.2402 & 0.8592 & 0.9995 & 0.9998 & 0.9357 & 0.8924 \\
\hline 9.4959 & 0.2600 & -1.7259 & 1.4826 & 1.9216 & 0.2647 & 0.6799 & 0.9988 & 0.9676 & 0.9596 & 0.9351 \\
\hline 15.071 & 0.1297 & -1.2783 & 1.5078 & 1.8124 & 0.2886 & 0.5768 & 0.9978 & 0.8899 & 0.9723 & 0.9567 \\
\hline 19.631 & 0.0802 & -1.0016 & 1.4968 & 1.8541 & 0.2782 & 0.6162 & 0.9983 & 0.7980 & 0.9675 & 0.9488 \\
\hline
\end{tabular}
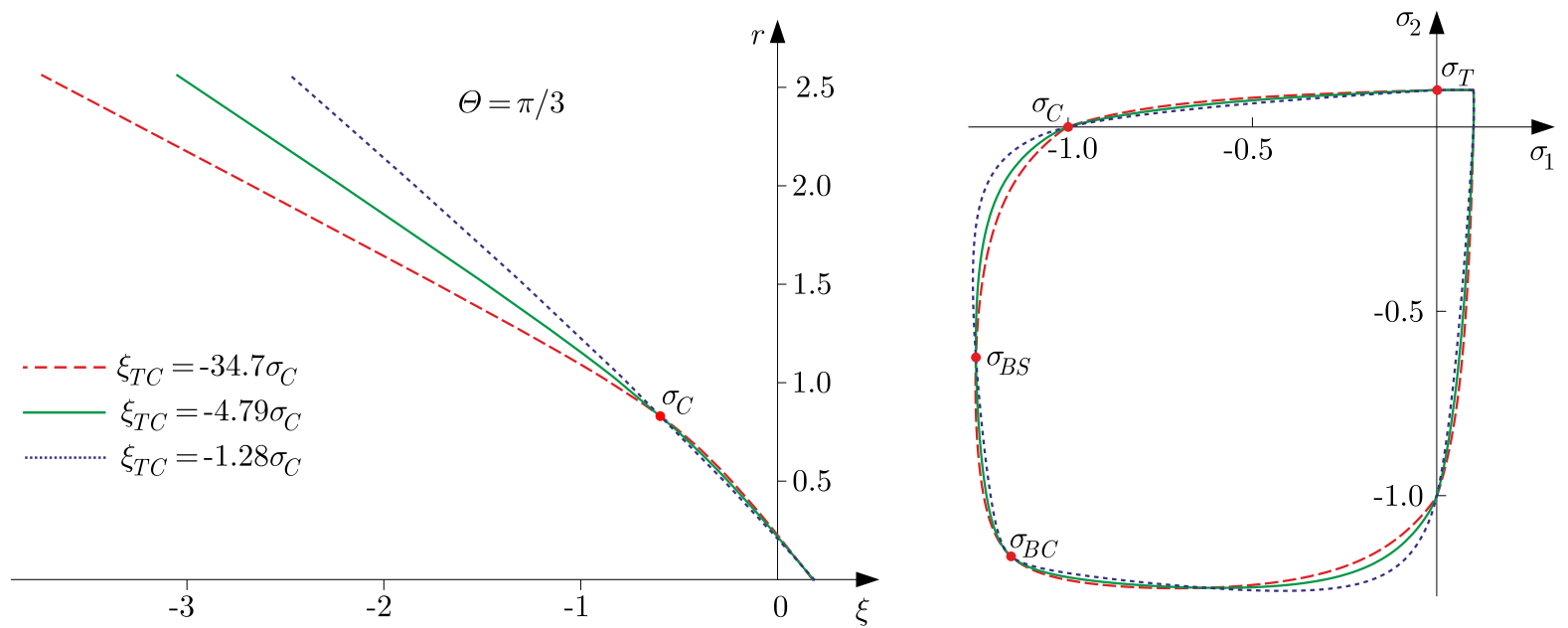

Fig. 7. The compressive meridian and the plane-stress cross-section of the yield surface for different locations of the triaxial compression test used for calibration: $\alpha_{T}=0.1, \alpha_{B C}=1.16, \alpha_{B S}=1.25$,

$$
B=0.2 \sigma_{C}
$$

Comparisons of the proposed criterion for the recommended calibration data with predictions of Lubliner's criterion and Podgórski's parabolic criterion are carried out. The deviatoric cross-sections of the surfaces are presented in Fig. 11, whereas the plane stress cross-sections are given in Fig. 12. 

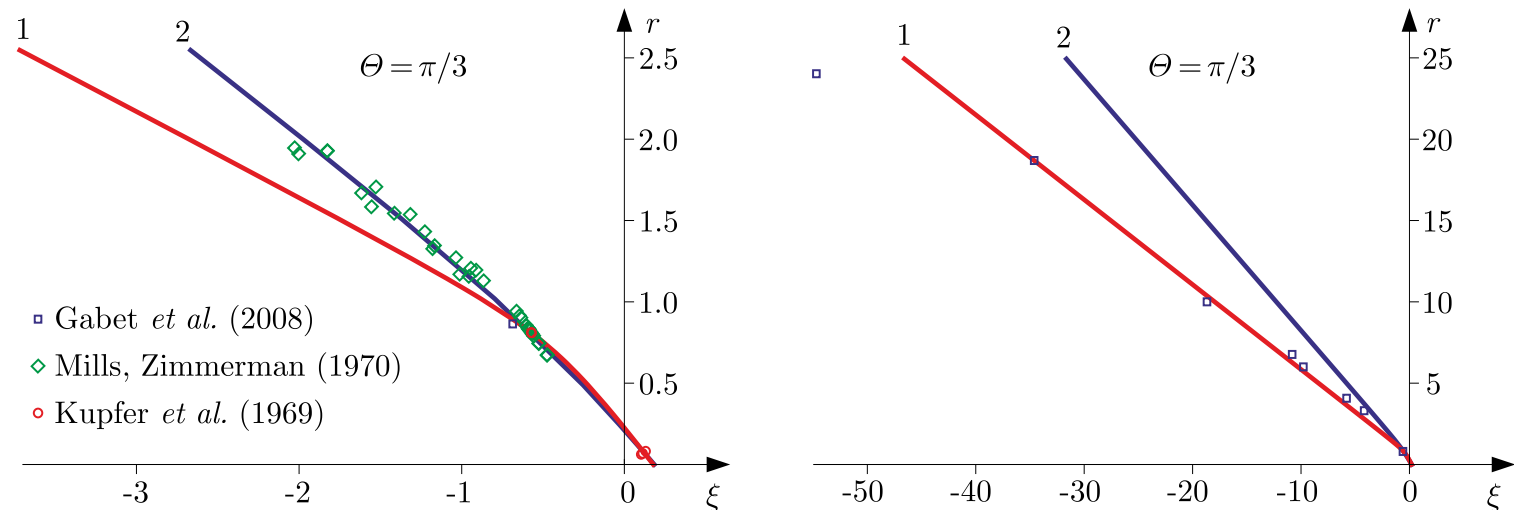

Fig. 8. The compressive meridians of the yield surface for: $\alpha_{T}=0.1, \alpha_{B C}=1.16, \alpha_{B S}=1.25$,

$B=0.2 \sigma_{C}$ and 1$\left.) \alpha_{T C}=12.3778, \eta=2.8555 ; 2\right) \alpha_{T C}=0.26, \eta=9.4976$ versus experimental data
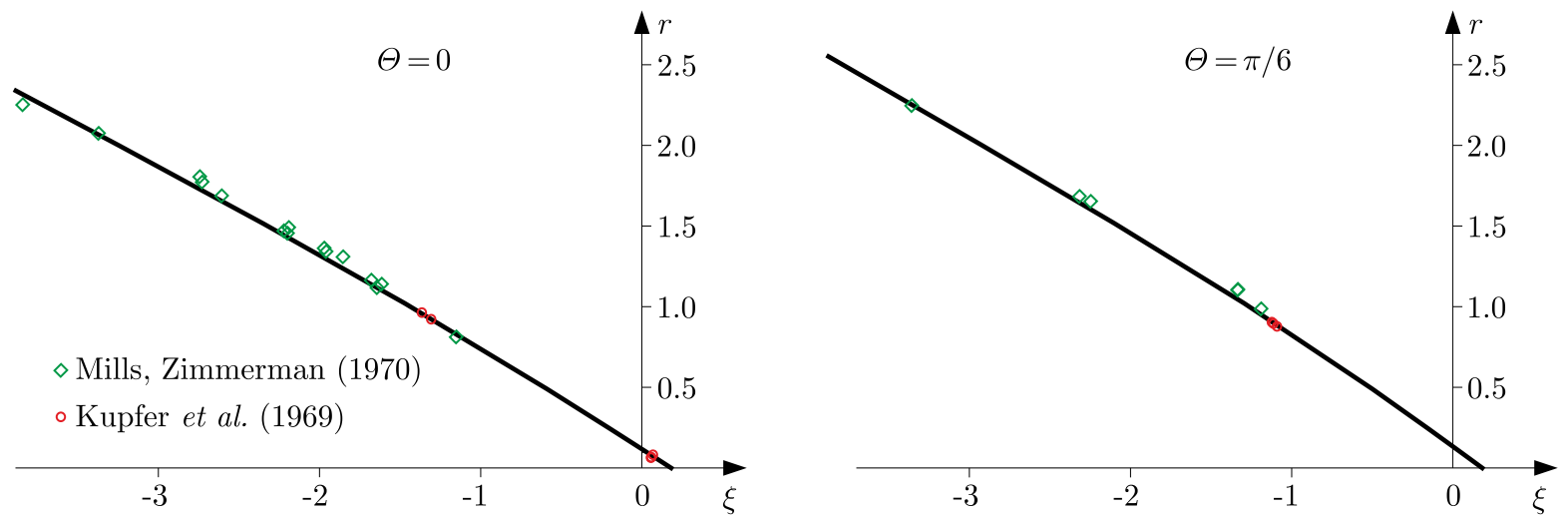

Fig. 9. The tensile and shear meridians of the yield surface for $\alpha_{T}=0.1, \alpha_{B C}=1.16, \alpha_{B S}=1.25$, $B=0.2 \sigma_{C}$ and $\alpha_{T C}=0.26, \eta=9.4976$ versus experimental data
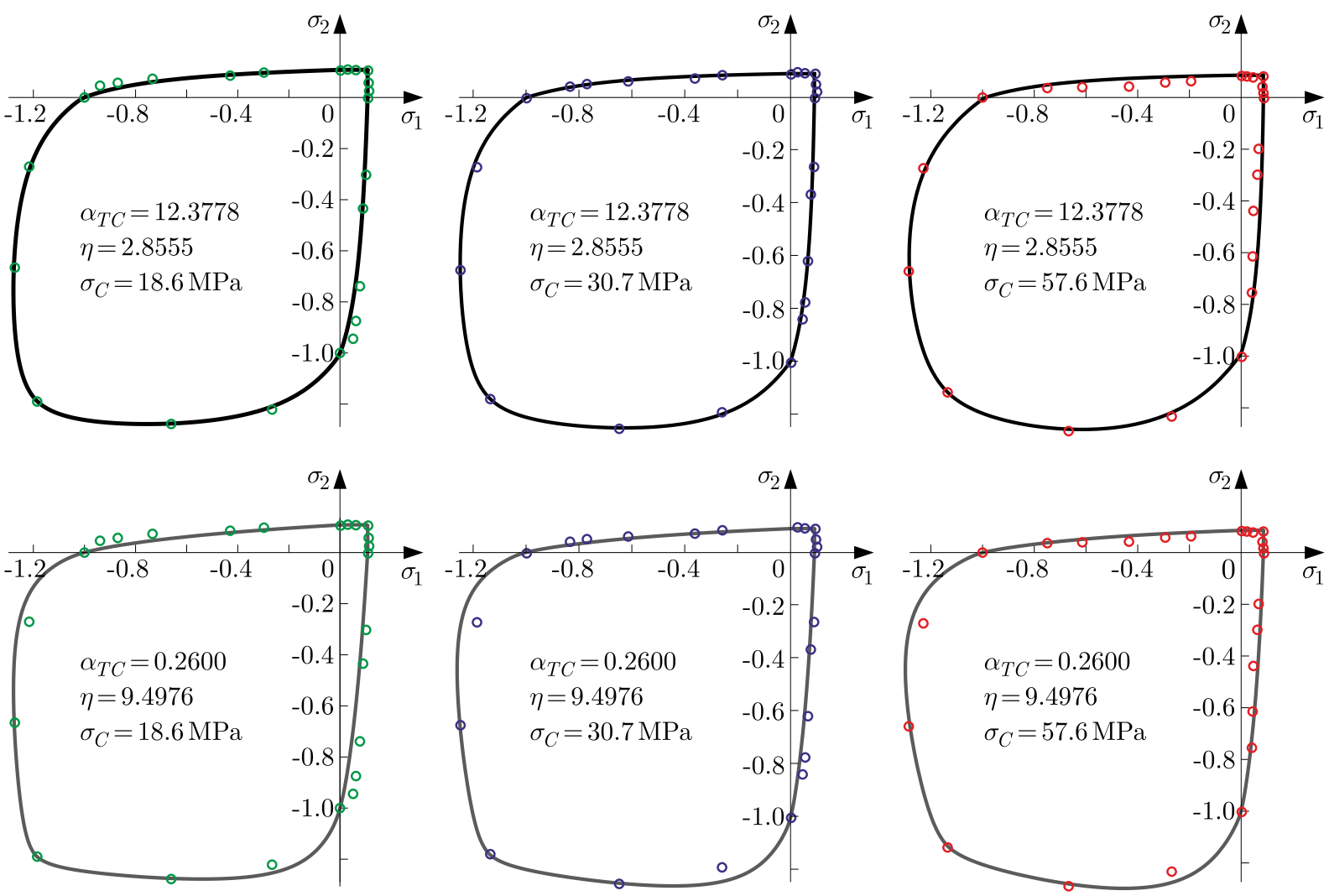

Fig. 10. Plane stress cross-sections versus experimental data (Kupfer et al., 1969) 
Curves of the deviatoric cross-sections indicate basic differences between the compared criteria. The proposed criterion exhibits almost a triangular section for a low confinement and a rounded hexagonal section for a higher hydrostatic pressure. The effect of regularisation of the Lubliner criterion is clearly seen for both singularities along the tensile and compressive meridians and the null maximum principal stress curve. Prediction of the Podgórski criterion results in more triangular deviatoric sections than the proposed.

In the case of the plane stress cross-sections, comparisons exhibit better accuracy of the proposed model than the Lubliner criterion and similar accuracy as the Podgórski parabolic criterion, see Fig. 10 and Fig. 12. The results for two sets of calibration data (recommended) are shown in Fig. 12. Depending on the selection of the location of the triaxial compression test, a different order of curves for the plane stress is reported when the proposed and Podgórski criteria predictions are plotted.
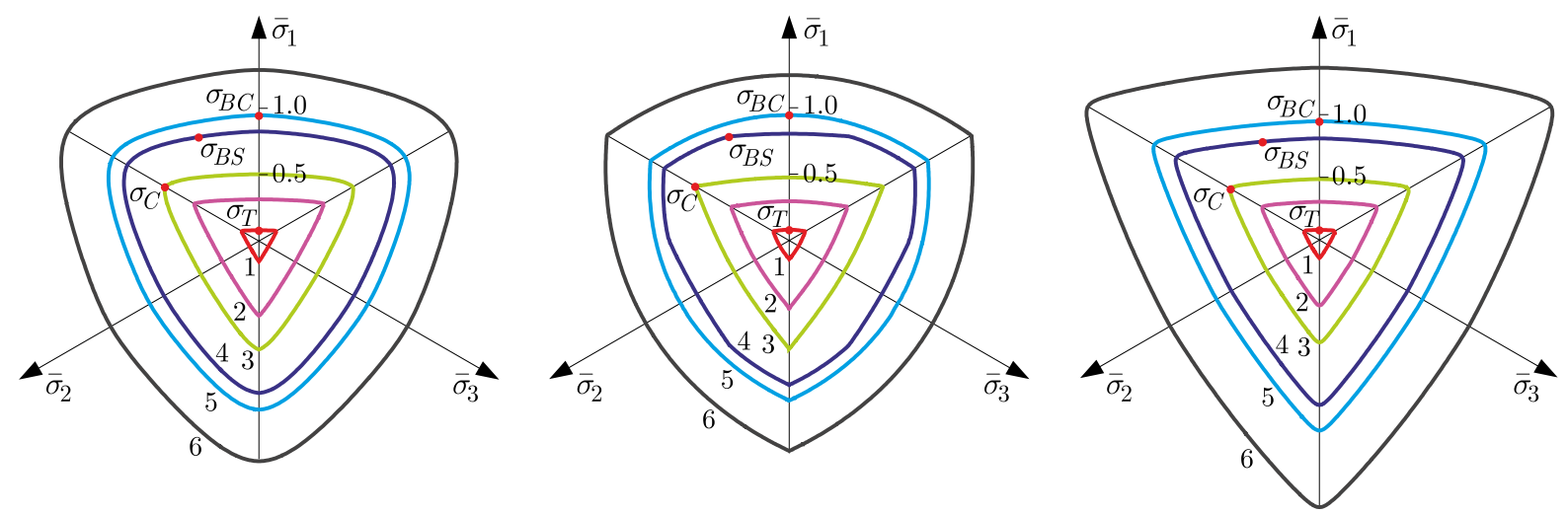

Fig. 11. Comparison of deviatoric cross-sections of the criterion (left) for $B=0.2 \sigma_{C}$ with the Lubliner criterion (central) and the Podgórski parabolic criterion (right): $\alpha_{T C}=6.722, \eta=2.835$, $\left.\left.\left.\left.\left.\left.\xi_{T C}=-18.73 \sigma_{C} .1\right) \xi=\xi_{T}, 2\right) 0.5 \xi_{C}, 3\right) \xi_{C}, 4\right) \xi_{B S}, 5\right) \xi_{B C}, 6\right) \xi_{T C} / 9 . \bar{\sigma}_{i}=\sigma_{i} \sqrt{2 / 3}$
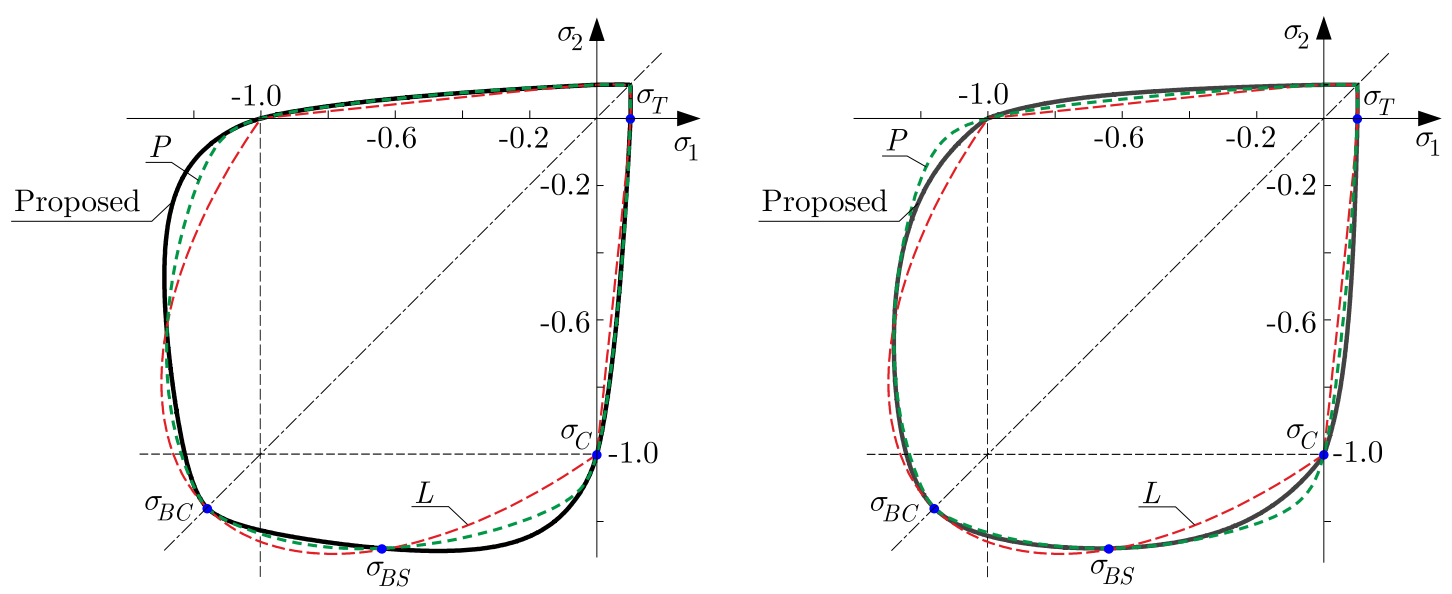

Fig. 12. Comparison of the criterion (proposed) for $B=0.2 \sigma_{C}$ with the criteria of Lubliner $(\mathrm{L})$ and Podgórski $(\mathrm{P})$ for the plane stress cross-section and for: $\alpha_{T C}=0.260$ and $\eta=9.498$ $\left(\xi_{T C}=-1.726 \sigma_{C}\right)(\mathrm{left}) ; \alpha_{T C}=6.722$ and $\eta=2.835\left(\xi_{T C}=-18.73 \sigma_{C}\right)$ (right)

The proposed yield function, (2.2), is quite general when it comes to the deviatoric crosssection shape. We have shown calibration for Podgórski functions (2.3), although any positive shape functions fulfilling (2.5) are appropriate. The choice of the Podgórski functions was dictated by its excellent properties - they are indefinitely differentiable and comply well with the experimental data for concrete as well as allow one to control the proportions of norms of devia- 
tors for three meridians (compressive, tensile and shear). If needed, the corners of the deviatoric cross-sections can be sharpened by using square roots of the Podgórski functions.

Function (2.2) can be used to describe yield initiation or failure not only for concrete, but also for various types of soils and rocks. For this reason, the shape functions can be chosen from an extensive range of proposals existing in the literature on the subject. Generally, they can be divided into two groups. The first are shape functions involving trigonometric functions of multiples of $3 \Theta$, which are most commonly used, including the Ottosen and Podgórski proposition, but also functions discussed by van Eekelen (1980), Ehlers (1995), Gudehus (1973), Argyris et al. (1974), Raniecki and Mróz (2008), Jemioło and Szwed (1999) among others. The second group are functions composed of segments connected at selected meridians, with the most recognised function of Willam and Warnke (1975) consisting of segments of an ellipse. The disadvantage of those shape functions is often the inability to find the first or the second derivative of the yield function in junction zones. Making use of an appropriate shape function can extend the range of application of the yield condition.

\section{Conclusions}

The presented modification of the Lubliner condition allows for a better adjustment of the yield surface to available experimental data than the original yield condition (Lubliner et al., 1989). The undeniable advantage is elimination of most singularities which are troublesome to deal with during structural analysis. Introduction of one free parameter $B$ into the proposed criterion removes singularity on the line of the null maximum principal stress reported in the Lubliner criterion, whereas the choice of appropriate shape functions allows one to smoothen the zones of compressive and tensile meridians. Additionally, the closed formulae describing material parameters facilitate the application of the criterion. The drawbacks of the proposed yield surface are limitations concerning convexity, although for typical data for concrete they are of no consequence. It was shown that using this criterion, one can improve the agreement of the prediction with available experimental results over a wide range of data. The second inconvenience is singularity at the apex that can be removed at the expense of adding an extra parameter, compare Szwed and Kamińska (2017).

Similarly as in the case of Lubliner criterion, different shape functions for deviatoric crosssections are used for the triaxial compression zone and for at least one positive principal stress zone. Between uniaxial and equal biaxial compression, there is a transition zone from one to another shape function, reflecting physical transition between failure modes. For the chosen shape functions, it is possible to calibrate the criterion analytically based on typical experimental tests: uniaxial tension and compression, equal biaxial compression, triaxial compression and pure shear located on compressive, tensile and shear meridians. The regularisation parameter is left undetermined to control smoothness of meridians (close to piecewise linear) in the zone of passing the null maximum principal stress curve. In total, depending on the shape function used, the yield condition involves seven or nine parameters.

The proposed plasticity surface exhibits desirable features reported in experiments. The meridians are appropriately curved, smooth and convex defining an open yield surface. The deviatoric cross-sections are smooth and convex being nearly triangular for a low confinement but becoming more circular (rounded hexagons) for increasing compressive stresses.

Multiple modifications of the Concrete Damaged Plasticity model of Abaqus are present in the relevant literature, usually postulating a novel description of damage. The proposed yield function could be easily incorporated into the model, improving its flexibility and reducing the difficulties connected to singularities as the original non-associative flow rule is already smooth. 


\section{References}

1. Abaqus 6.11 Theory Manual, 2011, Simulia

2. Argyris J.H., Faust G., Szimmat J., Warnke E.P., Willam K.J., 1974, Recent developments in the finite element analysis of prestressed concrete reactor vessels, Nuclear Engineering and Design, 28, 42-75, DOI: 10.1016/0029-5493(74)90088-0

3. Bigoni D., Piccolroaz A., 2004, Yield criteria for quasibrittle and frictional materials, International Journal of Solids and Structures, 41, 2855-2878, DOI: 10.1016/j.ijsolstr.2009.06.006

4. Drucker D.C., Prager W., 1952, Soil mechanics and plastic analysis or limit design, Quarterly of Applied Mechanics, 10, 157-165

5. Ehlers W., 1995, A single-surface yield function for geomaterials, Archives of Applied Mechanics, 65, 246-259, DOI: 10.1007/BF00805464

6. Gabet T., Malecot Y., Daudeville L., 2008, Triaxial behaviour of concrete under high stress: Influence of the loading path on compaction and limit states, Cement and Concrete Research, 38, 403-412, DOI: 10.1016/j.ijsolstr.2008.10.015

7. Gudehus G., 1973, Elastoplastic constitutive relations for sand (in German), Ingenieur-Archiv, 42, 151-169

8. Jemioło S., Szwed A., 1999, On application of convex functions to model failure of isotropic materials. Proposition of yield conditions for metals (in Polish), Prace Naukowe Politechniki Warszawskiej, 133, 5-51

9. Kupfer H., Hilsdorf H.K., Rusch H., 1969, Behavior of concrete under biaxial stresses, $A C I$ Journal, 66, 656-666

10. Lubliner J., Oliver J., Oller S., Onate E., 1989, A plastic-damage model for concrete, International Journal of Solids and Structures, 25, 299-326, DOI: 10.1016/0020-7683(89)90050-4

11. Mills L.L., Zimmerman R.M., 1970, Compressive strength of plain concrete under multiaxial loading conditions, ACI Journal, 67, 802-807

12. Ottosen N.S., 1977, A failure criterion for concrete, Journal of the Engineering Mechanics Division, 103, 527-535

13. Podgórski J., 1984, Limit state condition and dissipation function for isotropic materials, Archives of Mechanics, 36, 323-342

14. RANiecki B., Mróz Z., 2008, Yield or martensic phase transformation condition and dissipation functions for isotropic pressure insensitive alloys exhibiting SD effects, Acta Mechanica, 195, 81102, DOI: $10.1007 / \mathrm{s} 00707-007-0544-7$

15. Szwed A., Kamińska I., 2017, Modification of concrete damaged plasticity model. Part I: Modified plastic potential, MATEC Web of Conferences, 117, 00160, DOI: 10.1051/matecconf/201711700160

16. Szwed A., Kamińska I., 2019, Yield criteria for concrete built of deviatoric and meridional shape functions, [In:] Theoretical Foundations of Civil Engineering, IX, Mechanics of Materials and Structures, A. Szwed, I. Kamińska (Edit.), 85-94, Publishing House of Warsaw University of Technology

17. VAn Eekelen H.A.M., 1980, Isotropic yield surface in three dimensions for use in soil mechanics, International Journal for Numerical and Analytical Methods, 4, 89-101, DOI: 10.1002/nag.1610040107

18. Willam K.J., Warnke E.P., 1975, Constitutive models for triaxial behavior of concrete, Proceedings of the International Association for Bridge and Structural Engineering, 19, 1-30 